\title{
EXTREME STABILITY AND ALMOST PERIODICITY IN CONTINUOUS AND DISCRETE NEURONAL MODELS WITH FINITE DELAYS
}

\author{
S. MOHAMAD ${ }^{12}$ and K. GOPALSAMY ${ }^{3}$
}

(Received 18 June, 1999; revised 1 March, 2000)

\begin{abstract}
We consider the dynamical characteristics of a continuous-time isolated Hopfield-type neuron subjected to an almost periodic external stimulus. The model neuron is assumed to be dissipative having finite time delays in the process of encoding the external input stimulus and recalling the encoded pattern associated with the external stimulus. By using non-autonomous Halanay-type inequalities we obtain sufficient conditions for the hetero-associative stable encoding of temporally non-uniform stimuli. A brief study of a discrete-time model derived from the continuous-time system is given. It is shown that the discrete-time model preserves the stability conditions of the continuous-time system.
\end{abstract}

\section{Introduction}

The experimental and theoretical studies of Skarda and Freeman [28] and Freeman [11] suggest that a mammal's brain may be exploiting dynamic attractors for its encoding and subsequent associative recall of memories (or patterns) rather than temporally static (equilibrium-type) attractors as has been proposed in most studies of artificial neural networks. Limit cycles, strange attractors and other dynamical phenomena have been used by many authors to represent encoded temporal patterns as associative memories (Freeman et al. [12], Yau et al. [31], Chapeau-Blondeau and Chauvet [6], Moreira and Auto [25], Hjelmfelt and Ross [20]). Most of the existing literature on theoretical studies of artificial neural networks is predominantly concerned with

\footnotetext{
${ }^{1}$ On leave from Department of Mathematics, University Brunei Darussalam, Bandar Seri Begawan BE 1410, Brunei Darussalam; e-mail: sannay@ubd.edu.bn.

${ }^{2}$ Current address: Mathematics and Statistics, School of Informatics and Engineering, Flinders University of South Australia, Bedford Park SA 5042, Australia.

${ }^{3}$ Mathematics and Statistics, School of Informatics and Engineering, Flinders University of South Australia, Bedford Park SA 5042, Australia; e-mail: gopal@ist.flinders.edu.au.

(C) Australian Mathematical Society 2002, Serial-fee code 1446-1811/02
} 
autonomous systems containing temporally uniform network parameters and external input stimuli. Literature dealing with time-varying stimuli or network parameters appears to be scarce; such studies are however important to understand the dynamical characteristics of neuron behaviour in time varying environments.

It has been reported (see Eckhorn et al. [8], Gray et al. [18], Engel et al. [9]) that cell assemblies in the visual cortex oscillate synchronously in response to external stimuli. Such a synchrony is a manifestation of the encoding process of temporally-varying external stimuli. Ott et al. [26] have shown that one can convert a chaotic attractor to any one of a large number of possible time periodic motions by the introduction of time dependent perturbations of the network parameters.

In this article we study the dynamic behaviour of a neuron of Hopfield type in its process of encoding temporally non-uniform external stimulus and subsequent recalling of that encoded pattern. In particular we study the case where the external input stimulus is of almost periodic type. The neuron which oscillates synchronously in response to the time-varying external stimulus is assumed to be dissipative. In addition, we incorporate finite time delays in the processing part of the neuron's architecture. The incorporation of delays in the formulation is motivated by the following: delays are naturally present in biological networks through synaptic transmission, finite conduction velocity and neural processing of input stimuli. We refer to the articles of Gopalsamy and $\mathrm{He}[14,15]$ and the references therein for literature related to the stability of neural networks with time delays in temporally uniform environments modelled by autonomous delay and integro-differential equations.

The purpose of this article is to obtain sufficient conditions for the existence of a globally attractive almost periodic solution associated with an almost periodic external stimulus. In neuronal terminology, the globally attractive almost periodic solution represents the hetero-associative recall of the encoded pattern associated with the external input stimulus. The neuronal parameters, dissipation and gain, can either be temporally uniform, periodic or almost periodic. In other words the neuron being investigated has some synchronous manifestation in its encoding process of temporally-varying external stimuli. We consider the dynamics of artificial neuron models in continuous time and discrete-time domains with continuous states.

\section{Continuous-time-continuous-state model}

We study the dynamics of a single effective neuron subjected to a temporally non-uniform stimulus whose membrane potential $x(t)$ at time $t$ is modelled by a nonautonomous equation of the form (for more details see Caianiello and de Luca [5])

$$
\frac{d x(t)}{d t}=-a(t) x(t)+b(t) \tanh \left[\int_{0}^{t} K(s) x(t-s) d s\right]+c(t), \quad t>t_{0}
$$


where $\tau$ is a finite nonnegative constant, $K:[0, \tau] \mapsto[0, \infty)$ is assumed continuous and $0<\int_{0}^{\tau} K(s) d s<\infty, a(\cdot)$ denotes a dissipative or a negative feedback term, $b(\cdot)$ denotes the neuron gain and $c(\cdot)$ denotes the external stimulus. The real-valued functions $a(t), b(t)$ and $c(t)$ are continuous and are defined for $t \in \mathbb{R}$. They are assumed to satisfy the following:

$$
0<a_{*} \leq a(t) \leq a^{*}, \quad b_{*} \leq|b(t)| \leq b^{*}, \quad c_{*} \leq|c(t)| \leq c^{*}, \quad t \in \mathbb{B}
$$

where

$$
\begin{array}{lll}
a_{*}=\inf _{t \in \mathbb{R}} a(t), & b_{*}=\inf _{t \in \mathbb{R}}|b(t)|, & c_{*}=\inf _{t \in \mathbb{R}}|c(t)| \\
a^{*}=\sup _{t \in \mathbb{R}} a(t), & b^{*}=\sup _{t \in \mathbb{R}}|b(t)|, & c^{*}=\sup _{t \in \mathbb{R}}|c(t)| .
\end{array}
$$

We assume that (2.1) is supplemented with an initial condition of the form

$$
x(s)=\varphi(s), \quad s \in\left[t_{0}-\tau, t_{0}\right], \quad \varphi \in C\left(\left[t_{0}-\tau, t_{0}\right]\right)
$$

where $C\left(\left[t_{0}-\tau, t_{0}\right]\right)$ denotes the space of real-valued continuous functions defined on $\left[t_{0}-\tau, t_{0}\right]$ and $\sup _{s \in\left[t_{0}-\tau, t_{0}\right]}|\varphi(s)|<\infty$ for every $\varphi \in C\left(\left[t_{0}-\tau, t_{0}\right]\right)$. The following result establishes the boundedness of solutions of (2.1).

THEOREM 2.1. Suppose $a(t), b(t)$ and $c(t), t \in \mathbb{R}$, satisfy (2.2). Then

for $t>t_{0}$ and

$$
x\left(t_{0}\right) \in\left[-\frac{b^{*}+c^{*}}{a_{*}}, \frac{b^{*}+c^{*}}{a_{*}}\right] \Longrightarrow x(t) \in\left[-\frac{b^{*}+c^{*}}{a_{*}}, \frac{b^{*}+c^{*}}{a_{*}}\right]
$$

$$
|x(t)| \leq \frac{b^{*}+c^{*}}{a_{*}}+\sigma(t) \quad \text { for } t>t_{0} \quad \text { and } \quad \sigma(t) \rightarrow 0 \quad \text { as } t \rightarrow \infty .
$$

PROOF. By considering the upper right derivative $\frac{d^{+} \cdot 1}{d t}$ and the assumption (2.2) one obtains from (2.1) that $\frac{d^{+}}{d t}|x(t)| \leq-a_{*}|x(t)|+b^{*}+c^{*}, t>t_{0}$ from which we have

$$
|x(t)| \leq e^{-a_{*}\left(t-t_{0}\right)}\left(\left|x\left(t_{0}\right)\right|-\frac{b^{*}+c^{*}}{a_{*}}\right)+\frac{b^{*}+c^{*}}{a_{*}}, \quad t>t_{0} .
$$

Since $a_{*}>0$, both assertions (2.4) and (2.5) will follow from (2.6) and this completes the proof.

In the following we provide sufficient conditions for the extreme stability of (2.1). Let $x(t)=x(t, \varphi)$ and $y(t)=y(t, \psi), t>t_{0}$, denote arbitrary solutions of (2.1) corresponding to initial conditions $\varphi=\varphi(s)$ and $\psi=\psi(s)$ defined for $s \in\left[t_{0}-\tau, t_{0}\right]$ respectively. We assume that

$$
\sup _{s \in\left[t_{0}-\tau, t_{0}\right]}|\varphi(s)-\psi(s)|=M, \quad M>0 .
$$


Equation (2.1) is said to be extremely stable if $\lim _{t \rightarrow \infty}|x(t, \varphi)-y(t, \psi)|=0$, where $x(t, \varphi)$ and $y(t, \psi)$ denote arbitrary solutions of (2.1) corresponding to initial functions $\varphi, \psi \in C\left(\left[t_{0}-\tau, t_{0}\right]\right)$. The concept of extreme stability has been used in differential and delay differential equations and can be found in the work of LaSalle and Lefschetz [23]. The concept was then further extended by Yoshizawa [32]. Gopalsamy and his co-authors (see for instance Gopalsamy et al. [17], Gopalsamy and $\mathrm{He}$ [16]) have applied this stability in their investigations of non-autonomous delay logistic equations.

THEOREM 2.2. Let the assumption (2.2) hold. If there exists a real number $\mu>0$ such that

$$
a(t)-|b(t)| \int_{0}^{t} K(s) d s \geq \mu \quad \text { for } t \in \mathbb{R},
$$

then (2.1) is extremely stable.

PROOF. Since $x(t)=x(t, \varphi)$ and $y(t)=y(t, \psi)$ denote solutions of (2.1), we obtain

$$
\begin{aligned}
\frac{d}{d t}(x(t)-y(t))= & -a(t)(x(t)-y(t))+b(t) \tanh \left[\int_{0}^{\tau} K(s) x(t-s) d s\right] \\
& -b(t) \tanh \left[\int_{0}^{t} K(s) y(t-s) d s\right]
\end{aligned}
$$

for $t>t_{0}$. By considering the upper right derivative $\frac{d^{+}}{d t}$ and the mean value theorem of differential calculus, we derive from (2.9)

$$
\begin{aligned}
\frac{d^{+} v(t)}{d t} & \leq-a(t) v(t)+|b(t)| \int_{0}^{\tau} K(s) v(t-s) d s \\
& \leq-a(t) v(t)+|b(t)|\left(\int_{0}^{t} K(s) d s\right) \sup _{t-\tau \leq u \leq t} v(u), \quad t>t_{0},
\end{aligned}
$$

where $v(\cdot)=|x(\cdot)-y(\cdot)|$. We note that $0<\int_{0}^{\tau} K(s) d s<\infty$. The inequality (2.10) corresponds to a non-autonomous version of Halanay's inequality (Halanay [19, p. 378]). Halanay's inequality has been generalised to nonscalar systems of inequalities (for more details see Gopalsamy [13]) and to non-autonomous cases (see Baker and Tang [1]).

Let $\epsilon>1$ be arbitrary. We have from (2.7) that $v(t)<\epsilon M$ for $t \in\left[t_{0}-\tau, t_{0}\right]$. We claim

$$
v(t)<\epsilon M \text { for } t>t_{0}
$$


Suppose (2.11) does not hold. Let $t_{1}>t_{0}$ be the first instant for which

$$
v(t)<\epsilon M \quad \text { for } t_{0}-\tau \leq t<t_{1}, \quad v\left(t_{1}\right)=\epsilon M \quad \text { and } \quad \frac{d^{+} v\left(t_{1}\right)}{d t} \geq 0 .
$$

From (2.10) we have

$$
\frac{d^{+} v\left(t_{1}\right)}{d t} \leq-a\left(t_{1}\right) v\left(t_{1}\right)+\left|b\left(t_{1}\right)\right|\left(\int_{0}^{\tau} K(s) d s\right) \sup _{t_{1}-\tau \leq u \leq t_{1}} v(u)
$$

and by applying (2.8) and (2.12) to (2.13) we obtain

$$
\frac{d^{+} v\left(t_{1}\right)}{d t} \leq-\left(a\left(t_{1}\right)-\left|b\left(t_{1}\right)\right| \int_{0}^{t} K(s) d s\right) \epsilon M \leq-\mu \epsilon M<0 .
$$

From (2.12) and (2.14) we have a contradiction. Thus (2.11) holds. Since $\epsilon>1$ is arbitrary, by allowing $\epsilon \rightarrow 1^{+}$we have $v(t) \leq M$ for $t>t_{0}$.

Since $v(t)$ remains bounded for $t>t_{0}$ there exists a constant $\lambda$ such that

$$
\limsup _{t \rightarrow \infty} v(t)=\lambda
$$

and it follows from $0 \leq v(t) \leq M$ for $t>t_{0}$ that $0 \leq \lambda \leq M$. We wish to show $\lambda=0$. Suppose $\lambda>0$. We have $t-\tau \rightarrow \infty$ as $t \rightarrow \infty$ and it follows that

$$
\limsup _{t \rightarrow \infty}\left\{\sup _{t-\tau \leq u \leq t} v(u)\right\}=\lambda \text {. }
$$

By the assumption (2.8) there exists a constant $\delta$ satisfying $0<\delta<1$ such that

$$
|b(t)| \int_{0}^{\tau} K(s) d s<\delta a(t) \text { for } t \in \mathbb{R} .
$$

Let $\rho=\delta+(1-\delta) e^{-a_{*} T_{0}}$ where $T_{0}>t_{0}$ is a positive number. Since $a_{*}>0$, we have $0<\rho<1$. By applying the properties of limit superiors, it follows from (2.15) and (2.16) that for any $\zeta$ satisfying $0<\zeta \leq(1-\rho) \lambda /(1+\rho)$ there corresponds a sufficiently large positive number $T=T(\zeta)>T_{0}$ such that

$$
v(t)<\lambda+\zeta, \quad \sup _{t-\tau \leq u \leq t} v(u)<\lambda+\zeta \quad \text { for all } t \geq T .
$$

From (2.10) we have

$$
\frac{d^{+} v(u)}{d u} \leq-a(u) v(u)+|b(u)|\left(\int_{0}^{\tau} K(s) d s\right) \sup _{u-\tau \leq r \leq u} v(r)
$$

for $t-T \leq u \leq t, t \geq 2 T$ and hence

$$
\frac{d^{+}}{d u}\left(v(u) e^{-\int_{u}^{\prime} a(w) d w}\right) \leq|b(u)|\left(\int_{0}^{\tau} K(s) d s\right) \sup _{u-\tau \leq r \leq u} v(r) e^{-\int_{u}^{\prime} a(w) d w}
$$


for $t-T \leq u \leq t, t \geq 2 T$. Upon integrating (2.19) over the interval $u \in[t-T, t]$ for $t \geq 2 T$ we obtain

$$
\begin{aligned}
v(t) \leq & v(t-T) e^{-\int_{t-\tau}^{t} a(w) d w} \\
& +\int_{t-T}^{t}|b(u)|\left(\int_{0}^{\tau} K(s) d s\right) \sup _{u-\tau \leq r \leq u} v(r) e^{-\int_{u}^{t} a(w) d w} d u
\end{aligned}
$$

for $t \geq 2 T$. By applying (2.2), (2.17) and (2.18) we derive from (2.20)

$$
\begin{aligned}
v(t) & <(\lambda+\zeta) e^{-\int_{t-T}^{t} a_{*} d w}+\int_{t-T}^{t} \delta a(u)(\lambda+\zeta) e^{-\int_{u}^{\prime} a(w) d w} d u \\
& =(\lambda+\zeta) e^{-a_{*} T}+\delta(\lambda+\zeta) \int_{t-T}^{t} \frac{d}{d u}\left(e^{-\int_{u}^{\prime} a(w) d w}\right) d u \\
& \leq(\lambda+\zeta) e^{-a_{*} T}+\delta(\lambda+\zeta) \int_{t-T}^{t} a_{*} e^{-a_{*}(t-u)} d u \\
& =(\lambda+\zeta)\left(\delta+(1-\delta) e^{-a_{*} T}\right), \quad t \geq 2 T .
\end{aligned}
$$

Moreover, we also have from (2.15) (using a property of limit superiors) that there exists a sequence $\left\{t_{j}\right\}, j \in \mathbb{Z}^{+}$of nonnegative real numbers with $t_{j} \rightarrow \infty$ as $j \rightarrow \infty$ (if necessary we can take its subsequence) such that

$$
\lambda-\zeta<v\left(t_{j}\right) \text { for all } t_{j} \geq T_{1}=T_{1}(\zeta)
$$

where $T_{1}>T_{0}$ and $\zeta$ is chosen as in the above. From (2.21) and (2.22) we have

$$
\lambda-\zeta<v\left(t_{j}\right)<(\lambda+\zeta)\left(\delta+(1-\delta) e^{-a_{*} T}\right) \text { for all } t_{j} \geq T^{\prime}
$$

where $T^{\prime}=\max \left\{2 T, T_{1}\right\}$. It then follows that

$$
\lambda-\zeta<(\lambda+\zeta) \rho \Rightarrow \zeta>\frac{(1-\rho) \lambda}{1+\rho}
$$

and this contradicts our choice of $\zeta$. Hence $\lambda=0$. This in turn implies that

$$
\lim _{t \rightarrow \infty}|x(t)-y(t)|=0
$$

and hence (2.1) is extremely stable. This completes the proof.

We shall discuss the dynamics of (2.1) and its ability to encode external stimulus. In other words, the neuronal parameters $a(t)$ and $b(t)$ oscillate synchronously in response to the external stimulus $c(t)$. The dynamics of neural networks subjected to periodic inputs have been considered by Rescigno et al. [27], König and Schillen [22] and Bondarenko [3]. In this study, we investigate the dynamics of (2.1) when $a(t)$, 
$b(t)$ and $c(t)$ denote real-valued almost periodic functions defined for $t \in \mathbb{R}$. We refer to Besicovitch [2] and Corduneanu [7] for various properties of almost periodic functions; we refer to Fink [10] and Yoshizawa [33] for discussions on almost periodic differential and delay-differential equations. For the convenience of the reader we provide a formal definition of an almost periodic function $f(t), t \in \mathbb{R}$.

DEFINITION 2.1. A real-valued function $f(t), t \in \mathbb{R}$ is called almost periodic if for any given $\epsilon>0$ there corresponds a positive number $L=L(\epsilon)$ such that each interval of length $L$ on the real line contains at least one number $\mathfrak{p}=\mathfrak{p}(\epsilon)$ for which $\|f(t+\mathfrak{p})-f(t)\|<\epsilon$, where $\|f(t)\|=\sup _{t \in \mathbb{R}}|f(t)|$. The number $\mathfrak{p}$ is called the translation number or $\epsilon$-almost period of $f(t)$. The set $T(f, \epsilon)$ which contains the $\epsilon$-almost periods $\mathfrak{p}$ of $f(t)$ is relatively dense in $\mathbb{R}$.

We provide below a few properties of real-valued almost periodic functions for the benefit of the reader. The proofs of these properties can be found in the books mentioned above.

PROPERTY 2.1. An almost periodic function $f(t)$ is bounded and uniformly continuous for $t \in \mathbb{R}$.

PROPERTY 2.2. Let $f_{j}(t)$ be almost periodic for $t \in \mathbb{R}$ for each $j=1,2,3 \ldots$ If the sequence $\left\{f_{j}(t)\right\}$ converges to $f(t)$ as $j \rightarrow \infty$ uniformly for $t \in \mathbb{R}$ then the function $f(t)$ is almost periodic.

PROPERTY 2.3. Let $F(u)$ be a function uniformly continuous in the ball $\|u\|<A$ and let $f(t)$ be almost periodic such that $\|f(t)\|<A$ for $t \in \mathbb{R}$. Then the function $F(f(t))$ is almost periodic for $t \in \mathbb{R}$.

PROPERTY 2.4. A function $f(t)$ is almost periodic if and only if for any sequence of real numbers $\left\{m_{k}\right\}, k=1,2,3, \ldots$ there exists a subsequence $\left\{m_{k_{j}}\right\}, j=1,2,3, \ldots$ such that the sequence $\left\{f\left(t+m_{k_{j}}\right)\right\}$ converges as $j \rightarrow \infty$ uniformly for $t \in \mathbb{R}$.

PROPERTY 2.5. Let $f_{i}(t), i=1,2, \ldots, m$ denote almost periodic functions and $\epsilon>0$ be an arbitrary real number. Then there exists a positive real number $L=L(\epsilon)$ such that every interval of length $L$ contains at least one common $\epsilon$-almost period of $f_{i}(t)$ for all $i=1,2, \ldots, m$. The set $\bigcap_{i=1}^{m} T\left(f_{i}, \epsilon\right)$ is relatively dense in $\mathbb{R}$.

PROPERTY 2.6. Let $f(t)$ and $g(t)$ be almost periodic. Then $f(t) \pm g(t)$ and $f(t) g(t)$ are also almost periodic, and $f(t) / g(t)$ is almost periodic if $\inf _{t \in \mathbb{R}}|g(t)|=g_{*}>0$.

Let $(\mathscr{A} \mathscr{P},\|\cdot\|)$ denote the space of all real-valued almost periodic functions defined on $\mathbb{R}$ with $\|\cdot\|$ denoting the supremum norm. By Property 2.1 , each element in $\mathscr{A} \mathscr{P}$ 
is bounded in the norm $\|\cdot\|$. By Property 2.6 , we deduce that $(\mathscr{A} \mathscr{P},\|\cdot\|)$ forms a linear space with respect to addition of almost periodic functions and multiplication by scalars. Due to Property 2.2 , the topology of the norm $\|\cdot\|$ is equivalent to the topology of uniform convergence on $\mathbb{R}$ of sequences of almost periodic functions. Hence $(\mathscr{A} \mathscr{P},\|\cdot\|)$ is a complete normed space and thus a Banach space.

In the following we prove the existence of a unique almost periodic solution of (2.1) which is globally attractive. We recall a well-known fixed point theorem (see Istratescu [21]) of the following form:

SCHAUDER'S FIXED POINT THEOREM. Let $\mathscr{M}$ denote a compact convex subset of a Banach space and let $P: \mathscr{M} \rightarrow \mathscr{M}$ be continuous. Then the mapping $P$ has a fixed point in $\mathscr{M}$.

THEOREM 2.3. Let $a(t), b(t), c(t) \in \mathscr{A} \mathscr{P}$ be defined for $t \in \mathbb{R}$ and assume they satisfy (2.2). Suppose (2.8) holds. Then there exists a unique almost periodic solution $x^{*}(t)$ of (2.1) which is globally attractive.

PROOF. We define a set $\mathscr{B} \subset \mathscr{A} \mathscr{P}$ such that every $\phi(t) \in \mathscr{B}, t \in \mathbb{R}$ satisfies

$$
\begin{aligned}
\|\phi(t)\| & \leq\left(b^{*}+c^{*}\right) / a_{*} \quad \text { and } \\
\|\phi(t+\mathfrak{p})-\phi(t)\| & \leq \epsilon, \quad \mathfrak{p}=\mathfrak{p}(\epsilon) \quad \text { for any } \epsilon>0 .
\end{aligned}
$$

From (2.24) and (2.25), it follows that the set $\mathscr{B}$ is uniformly bounded and equialmost periodic. The requirement (2.25) implies that for any $\epsilon>0$ there corresponds a positive number $L=L(\epsilon)$ such that every interval of length $L$ contains at least one common $\epsilon$-almost period $\mathfrak{p}=\mathfrak{p}(\epsilon)$ of every element $\phi(\cdot) \in \mathscr{B}$. The convexity of the set $\mathscr{B}$ can be verified. We show the compactness of $\mathscr{B}$ as follows: let $\left\{\phi_{i}(t)\right\}, t \in \mathbb{R}$, $i \in \mathbb{Z}^{+}=\{1,2,3, \ldots\}$ denote an arbitrary sequence from the set $\mathscr{B}$. By (2.24), we have

$$
\left\|\phi_{i}(t)\right\| \leq\left(b^{*}+c^{*}\right) / a_{*} \text { for } i \in \mathbb{Z}^{+} .
$$

Using the equi-almost periodicity of $\left\{\phi_{i}(t)\right\}$, there exists a positive number $L=L(\epsilon / 3)$ such that every interval of length $L$ contains at least one number $\mathfrak{p}=\mathfrak{p}(\epsilon / 3)$ for which

$$
\left\|\phi_{i}(t+\mathfrak{p})-\phi_{i}(t)\right\| \leq \epsilon / 3, \quad i \in \mathbb{Z}^{+} .
$$

To show that the set $\mathscr{B}$ is compact we must show that there exists a subsequence of $\left\{\phi_{i}(t)\right\}$ which is convergent in $\mathscr{B}$ for all $t \in \mathbb{R}$. Since the set $\left\{\phi_{i}(t)\right\}$ is equi-continuous on an interval of length $L=L(\epsilon / 3)$ and the set satisfies (2.26), by the Arzela-Ascoli theorem there exists a subsequence $\left\{\phi_{i_{\mu}}(t)\right\}, \mu \in \mathbb{Z}^{+}$such that

$$
\sup _{t \in[0, L]}\left|\phi_{i_{\mu}}(t)-\phi_{i_{\nu}}(t)\right| \leq \epsilon / 3 \text { for all } \mu, \nu \geq l=l(\epsilon / 3) .
$$


Choose any $s \in \mathbb{R}$. Since $\mathfrak{p} \in[-s,-s+L]$ we must have $\mathfrak{p}+s \in[0, L]$. By applying (2.27), (2.28) and the triangle inequality we derive that

$$
\begin{aligned}
\sup _{s \in \mathbb{R}}\left|\phi_{i_{\mu}}(s)-\phi_{i_{\nu}}(s)\right| \leq & \sup _{s \in \mathbb{R}}\left|\phi_{i_{\mu}}(s)-\phi_{i_{\mu}}(s+\mathfrak{p})\right|+\sup _{s+\mathfrak{p} \in[0, L]}\left|\phi_{i_{\mu}}(s+\mathfrak{p})-\phi_{i_{\nu}}(s+\mathfrak{p})\right| \\
& +\sup _{s \in \mathbb{R}}\left|\phi_{i_{v}}(s+\mathfrak{p})-\phi_{i_{v}}(s)\right| \\
\leq & \epsilon / 3+\epsilon / 3+\epsilon / 3=\epsilon \quad \text { for all } \mu, v \geq l .
\end{aligned}
$$

Since $s \in \mathbb{R}$ is arbitrary we have from (2.29) that the subsequence $\left\{\phi_{i_{\mu}}(s)\right\}$ converges for all $s \in \mathbb{R}$. This in turn implies that the set $\mathscr{B}$ has compact closure in the topology of uniform convergence and hence the set $\mathscr{B}$ is compact.

Now let us define a mapping $P$ by

$$
\begin{aligned}
(P \phi)(t)= & \int_{0}^{\infty}\left(b(t-r) \tanh \left[\int_{0}^{\tau} K(u) \phi(t-r-u) d u\right]+c(t-r)\right) \\
& \times e^{-\int_{0}^{\prime} a(t-s) d s} d r
\end{aligned}
$$

for $\phi(t) \in \mathscr{B}, t \in \mathbb{R}$. By applying (2.2) one obtains from (2.30) that

$$
\|(P \phi)(t)\| \leq\left(b^{*}+c^{*}\right) \int_{0}^{\infty} e^{-\int_{0}^{r} a_{*} d s} d r=\frac{b^{*}+c^{*}}{a_{*}}
$$

for $\phi(t) \in \mathscr{B}, t \in \mathbb{R}$. Let $\epsilon>0$ be arbitrary. Choose $\epsilon^{*}$ such that

$$
\epsilon^{*}=\min \left\{\frac{\epsilon}{4} \frac{a_{*}^{2}}{b^{*}+c^{*}}, \frac{\epsilon}{4} a_{*}, \frac{\epsilon}{4 \int_{0}^{\tau} K(u) d u} \frac{a_{*}}{b^{*}}\right\} .
$$

We note that $0<\int_{0}^{\tau} K(u) d u<\infty$. For this $\epsilon^{*}$, by Property 2.5 , there exists a common almost period $\mathfrak{p}=\mathfrak{p}\left(\epsilon^{*}\right)$ of $a(\cdot), b(\cdot), c(\cdot), \phi(\cdot)$, namely

$$
\begin{aligned}
|a(t+\mathfrak{p}-s)-a(t-s)| & \leq \epsilon^{*} \leq \frac{\epsilon}{4} \frac{a_{*}^{2}}{b^{*}+c^{*}}, \\
|b(t+\mathfrak{p}-r)-b(t-r)| & \leq \epsilon^{*} \leq \frac{\epsilon}{4} a_{*}, \\
|c(t+\mathfrak{p}-r)-c(t-r)| & \leq \epsilon^{*} \leq \frac{\epsilon}{4} a_{*}, \\
|\phi(t+\mathfrak{p}-r-u)-\phi(t-r-u)| & \leq \epsilon^{*} \leq \frac{\epsilon}{4 \int_{0}^{\tau} K(u) d u} \frac{a_{*}}{b^{*}},
\end{aligned}
$$

for $r, s, t, u \in \mathbb{R}$. From (2.30) we estimate $|(P \phi)(t+\mathfrak{p})-(P \phi)(t)|$ as follows:

$$
|(P \phi)(t+\mathfrak{p})-(P \phi)(t)|
$$




$$
\begin{aligned}
\leq & \int_{0}^{\infty}|b(t+\mathfrak{p}-r)-b(t-r)|\left|\tanh \left(\int_{0}^{\tau} K(u) \phi(t+\mathfrak{p}-r-u) d u\right)\right| \\
& \times e^{-\int_{0}^{r} a(t+\mathfrak{p}-s) d s} d r \\
& +\int_{0}^{\infty}|b(t-r)| \mid \tanh \left(\int_{0}^{\tau} K(u) \phi(t+\mathfrak{p}-r-u) d u\right) \\
& +\int_{0}^{\infty}|b(t-r)|\left|\tanh \left(\int_{0}^{\tau} K(u) \phi(t-r-u) d u\right)\right| \\
& \times\left|e^{-\int_{0}^{r} a(t+\mathfrak{p}-s) d s}-e^{-\int_{0}^{r} a(t-s) d s}\right| d r \\
& +\int_{0}^{\infty}|c(t+\mathfrak{p}-r)-c(t-r)| e^{-\int_{0}^{r} a(t-s) d s} d r \\
& +\int_{0}^{\infty}|c(t+\mathfrak{p}-r)|\left|e^{-\int_{0}^{r} a(t+\mathfrak{p}-s) d s}-e^{-\int_{0}^{r} a(t-s) d s}\right| d r
\end{aligned}
$$

for $\phi(t) \in \mathscr{B}, t \in \mathbb{R}$. By using the mean value theorem of differential calculus and (2.32) we can show the following:

$$
\begin{aligned}
& \left|\tanh \left(\int_{0}^{\tau} K(u) \phi(t+\mathfrak{p}-r-u) d u\right)-\tanh \left(\int_{0}^{\tau} K(u) \phi(t-r-u) d u\right)\right| \\
& \quad=\left|\operatorname{sech}^{2}(\theta)\left(\int_{0}^{\tau} K(u) \phi(t+\mathfrak{p}-r-u) d u-\int_{0}^{\tau} K(u) \phi(t-r-u) d u\right)\right| \\
& \quad \leq \int_{0}^{\tau} K(u)|\phi(t+\mathfrak{p}-r-u)-\phi(t-r-u)| d u \leq \frac{\epsilon}{4} \frac{a_{*}}{b^{*}}
\end{aligned}
$$

where $\theta$ lies between $\int_{0}^{\tau} K(u) \phi(t+\mathfrak{p}-r-u) d u$ and $\int_{0}^{\tau} K(u) \phi(t-r-u) d u$. Similarly,

$$
\begin{aligned}
\left|e^{-\int_{0}^{r} a(t+\mathfrak{p}-s) d s}-e^{-\int_{0}^{\prime} a(t-s) d s}\right| & =\left|e^{\theta^{\prime}}\left(-\int_{0}^{r} a(t+\mathfrak{p}-s) d s+\int_{0}^{r} a(t-s) d s\right)\right| \\
& \leq e^{-\int_{0}^{\prime} a_{*} d s} \int_{0}^{r}|a(t+\mathfrak{p}-s)-a(t-s)| d s \\
& \leq e^{-a_{*} r} \frac{\epsilon}{4} \frac{r a_{*}^{2}}{b^{*}+c^{*}}
\end{aligned}
$$

where $\theta^{\prime}$ lies between $-\int_{0}^{r} a(t+\mathfrak{p}-s) d s$ and $-\int_{0}^{r} a(t-s) d s$. We note that

$$
-\int_{0}^{r} a^{*} d s \leq \theta^{\prime} \leq-\int_{0}^{r} a_{*} d s
$$


We use (2.32), (2.34) and (2.35) in (2.33) to derive

$$
\begin{aligned}
|(P \phi)(t+\mathfrak{p})-(P \phi)(t)| \leq & \frac{\epsilon}{4} a_{*} \int_{0}^{\infty} e^{-\int_{0}^{r} a_{*} d s} d r+b^{*} \frac{\epsilon}{4} \frac{a_{*}}{b^{*}} \int_{0}^{\infty} e^{-\int_{0}^{r} a_{*} d s} d r \\
& +b^{*} \frac{\epsilon}{4} \frac{a_{*}^{2}}{b^{*}+c^{*}} \int_{0}^{\infty} r e^{-a_{*} r} d r+\frac{\epsilon}{4} a_{*} \int_{0}^{\infty} e^{-\int_{0}^{r} a_{*} d s} d r \\
& +c^{*} \frac{\epsilon}{4} \frac{a_{*}^{2}}{b^{*}+c^{*}} \int_{0}^{\infty} r e^{-a_{*} r} d r=\epsilon
\end{aligned}
$$

for $\phi(t) \in \mathscr{B}, t \in \mathbb{R}$. One can see that the common almost period $\mathfrak{p}$ which satisfies (2.32) is actually an $\epsilon$-almost period of $(P \phi)(t)$. By (2.31) and (2.36) we deduce that $\phi(t) \in \mathscr{B}$ implies $(P \phi)(t) \in \mathscr{B}$.

Next, we let $\epsilon^{\prime}>0$ denote an arbitrary real number. We take any two $\phi_{1}, \phi_{2} \in \mathscr{B}$ such that $\left\|\phi_{1}(t)-\phi_{2}(t)\right\|<\delta$, where $\delta=\epsilon^{\prime} a_{*} /\left(b^{*} \int_{0}^{\tau} K(u) d u\right)$. From (2.30) we obtain

$$
\begin{aligned}
\left(P \phi_{1}\right)(t)-\left(P \phi_{2}\right)(t)= & \int_{0}^{\infty} b(t-r)\left(\tanh \left[\int_{0}^{t} K(u) \phi_{1}(t-r-u) d u\right]\right. \\
& \left.-\tanh \left[\int_{0}^{\tau} K(u) \phi_{2}(t-r-u) d u\right]\right) e^{-\int_{0}^{r} a(t-s) d s} d r
\end{aligned}
$$

and it will follow that

$$
\left\|\left(P \phi_{1}\right)(t)-\left(P \phi_{2}\right)(t)\right\|<b^{*}\left(\int_{0}^{\tau} K(u) d u\right) \delta \int_{0}^{\infty} e^{-\int_{0}^{r} a_{*} d s} d r=\epsilon^{\prime} .
$$

This in turn implies that the mapping $P$ is continuous with respect to $\phi(t) \in \mathscr{B}$, $t \in \mathbb{R}$. We have that $\mathscr{B}$ is a compact convex subset of the Banach space $\mathscr{A} \mathscr{P}$ and $P: \mathscr{B} \rightarrow \mathscr{B}$ is continuous. By Schauder's fixed point theorem, there exists at least one $x^{*} \in \mathscr{B}$ such that $P x^{*}=x^{*}$.

Next we show that $x^{*}(t)$ defined for $t \in \mathbb{R}$ is a solution of (2.1). We have

$$
\begin{aligned}
\frac{d x^{*}(t)}{d t}=\frac{d}{d t} & \left\{\int _ { 0 } ^ { \infty } \left(b(t-r) \tanh \left[\int_{0}^{\tau} K(u) x^{*}(t-r-u) d u\right]\right.\right. \\
& \left.+c(t-r)) e^{-\int_{0}^{r} a(t-s) d s} d r\right\} \\
= & \frac{d}{d t} \int_{-\infty}^{t}\left(b(r) \tanh \left[\int_{0}^{\tau} K(u) x^{*}(r-u) d u\right]+c(r)\right) e^{-\int_{r}^{*} a(s) d s} d r \\
= & -a(t) x^{*}(t)+b(t) \tanh \left[\int_{0}^{\tau} K(u) x^{*}(t-u) d u\right]+c(t), \quad t \in \mathbb{R}
\end{aligned}
$$

and hence $x^{*}(t)$ is an almost periodic solution of (2.1) for $t \in \mathbb{R}$. This $x^{*}(t)$ is a nontrivial solution if $c(t)$ is nontrivial. 


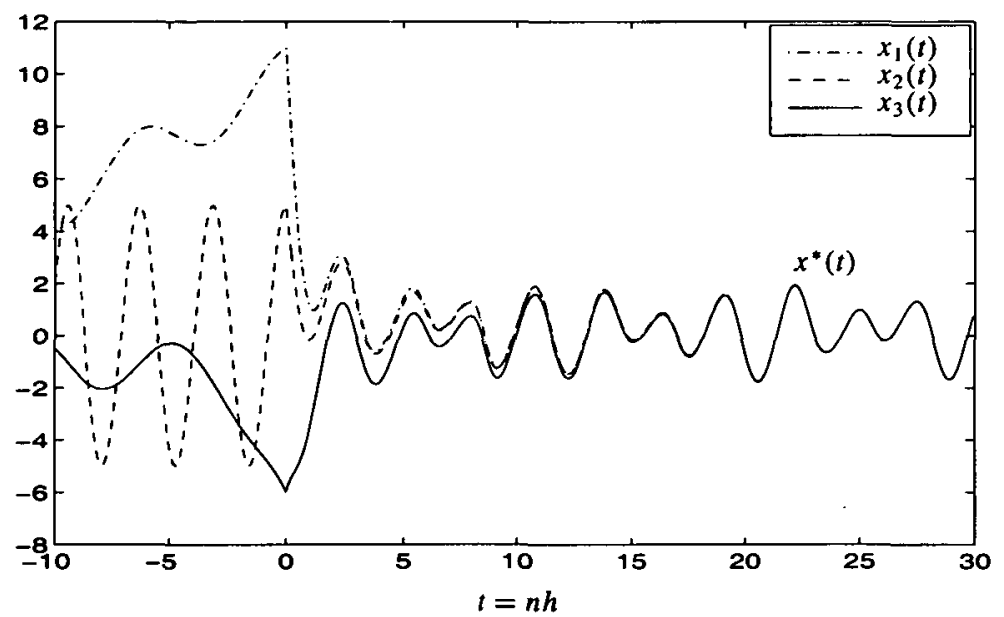

FIGURE 1. Three neuronal states $x_{1}(t), x_{2}(t)$ and $x_{3}(t)$ converge towards the globally attractive almost periodic encoded pattern $x^{*}(t)$. The existence of $x^{*}(t)$ can be seen for $t \geq 20$.

Now let $x(t, \varphi) \in L^{\infty}$ be an arbitrary solution of $(2.1)$; let $\left(L^{\infty},\|\cdot\|\right)$ denote the space of bounded continuous functions defined on $\mathbb{R}$. We have from Theorem 2.3 that $\lim _{t \rightarrow \infty}\left|x(t, \varphi)-x^{*}(t)\right|=0$ which implies the global attractivity of the solution $x^{*}(t)$. The uniqueness of $x^{*}(t)$ follows from its nature being almost periodic and globally attractive. This completes the proof.

COROLLARY 2.4. Suppose the condition (2.2) holds and let $\omega>0$ be the common period of $a(t), b(t)$ and $c(t)$, that is,

$$
a(t+\omega)=a(t), \quad b(t+\omega)=b(t), \quad c(t+\omega)=c(t) \quad \text { for } t \in \mathbb{R} .
$$

If the condition (2.8) holds then there exists a unique $\omega$-periodic solution $x_{\omega}^{*}(t)$ of (2.1) which is globally attractive.

PROOF. Let $\phi \in \mathscr{B}_{\omega}$ be such that $\phi(t+\omega)=\phi(t)$ for all $t \in \mathbb{R}$ and

$$
\sup _{t \in[0, \omega]}|\phi(t)| \leq\left(b^{*}+c^{*}\right) / a_{*} .
$$

The set $\mathscr{B}_{\omega}$ is a compact convex subset of the Banach space of all $\omega$-periodic functions. By using the mapping $P$ given by (2.30) and by applying the periodicity of $a(\cdot), b(\cdot)$, $c(\cdot)$ and $\phi(\cdot)$ in (2.33) one can show that $(P \phi)(t+\omega)=(P \phi)(t)$ for all $t \in \mathbb{R}$. The rest of the proof follows similarly as in the proof of Theorem 2.3. This completes the proof. 


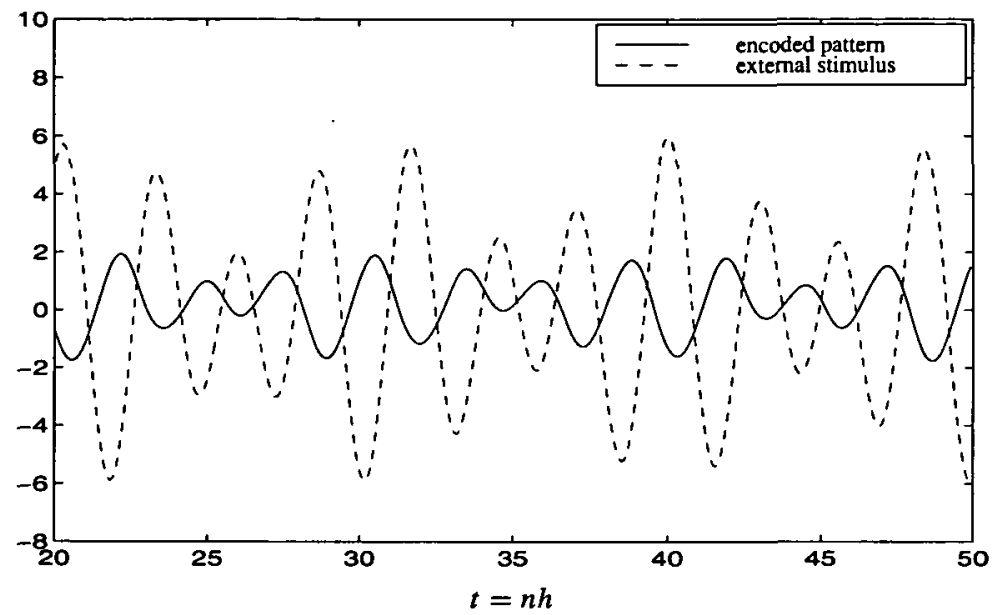

FIGURE 2. The encoded pattern $x^{*}(t)$ associated with the external stimulus $c(t)$ is illustrated for time $20 \leq t \leq 50$.

EXAMPLE 2.1. We provide computer simulations of the neuronal model (2.1) for $t>0$ to illustrate the existence of a unique globally attractive encoded pattern $x^{*}(t)$ associated with the almost periodic external stimulus, $c(t)$. For the simulations we assume the following:

$$
\begin{aligned}
\tau & =10 ; \quad K(s)=e^{-s}, \quad s \in[-\tau, 0] ; & & \\
a(t) & =2.0+0.6 \cos (\sqrt{5} t)+0.4 \sin (\pi t / 2), & & \text { almost periodic; } \\
b(t) & =1.6+0.6 \cos (\sqrt{5} t)+0.4 \sin (\pi t / 2), & & \text { almost periodic; } \\
c(t) & =4.0 \sin (\sqrt{5} t)+2.0 \cos (\pi t / 2), & & \text { almost periodic. }
\end{aligned}
$$

In simulating (2.1), we use a numerical scheme of the form

$$
\begin{aligned}
x((n+1) h)= & \frac{1}{1+a(n h) h} x(n h)+\frac{b(n h) h}{1+a(n h) h} \tanh \left[\sum_{j=1}^{\kappa} \tilde{K}(j h) x((n-j) h)\right] \\
& +\frac{c(n h) h}{1+a(n h) h}, \quad n=0,1,2, \ldots,
\end{aligned}
$$

where

$$
h=0.1, \quad \kappa=100, \quad \tilde{K}(j h)=\frac{1-e^{-h}}{e^{-h}} e^{-j h}, \quad j=1,2, \ldots, \kappa .
$$

We note from the above that $\int_{0}^{\tau} K(s) d s=\sum_{j=1}^{\kappa} \tilde{K}(j h)$. The derivation and the dynamical characteristics of the numerical scheme are discussed in the following 


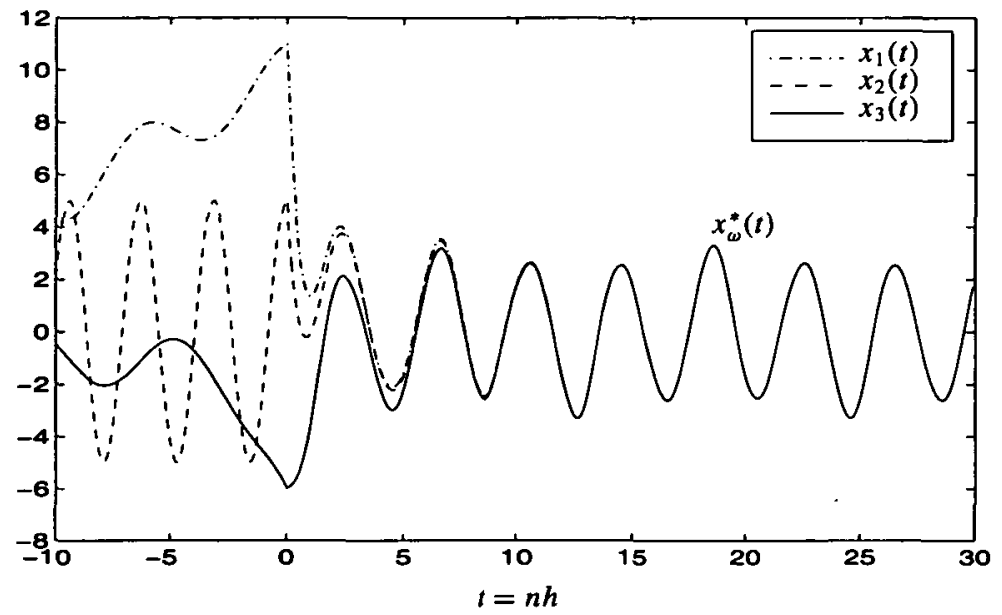

FIGURE 3. Three neuronal states $x_{1}(t), x_{2}(t)$ and $x_{3}(t)$ converge towards the globally attractive $\omega$-periodic encoded pattern $x_{\omega}^{*}(t)$. The existence of $x_{\omega}^{*}(t)$ can be seen for $t \geq 15$.

section. Figure 1 illustrates three solutions $x_{1}(t), x_{2}(t)$ and $x_{3}(t)$ converging towards the attractive encoded pattern $x^{*}(t)$. The initial values of the three solutions for $s \in[-\tau, 0]$ are provided by

$$
\begin{aligned}
& x_{1}(s)=\tau+0.5 s+\cos (s), \\
& x_{2}(s)=5 \cos (2 s), \\
& x_{3}(s)=-1-5 e^{0.6 s}+\sin (s) .
\end{aligned}
$$

In Figure 2, we provide a plot of the encoded pattern $x^{*}(t)$ and the external stimulus, $c(t)$.

EXAMPLE 2.2. We illustrate the existence of a unique globally attractive $\omega$-periodic encoded pattern $x_{\omega}^{*}(t)$ of (2.1). For the computer simulations we assume the following:

$$
\begin{aligned}
\tau & =10 ; \quad K(s)=e^{-s}, & & s \in[-\tau, 0] ; \\
a(t) & =2.0+0.4 \sin (\pi t / 3), & & \text { period }=6 ; \\
b(t) & =1.6+0.4 \sin (\pi t / 3), & & \text { period }=6 ; \\
c(t) & =8.0 \cos (\pi t / 2), & & \text { period }=4 .
\end{aligned}
$$

The common period of $a(t), b(t)$ and $c(t)$ is $\omega=12$. Again we use the numerical scheme (2.38) in simulating (2.1). Figure 3 illustrates three neuronal states $x_{1}(t), x_{2}(t)$ and $x_{3}(t)$ converging towards the $\omega$-periodic encoded pattern $x_{\omega}^{*}(t)$. The initial values of the three neuronal states are provided by (2.39). A plot of the encoded pattern $x_{\omega}^{*}(t)$ and the external stimulus $c(t)$ is provided by Figure 4 . 


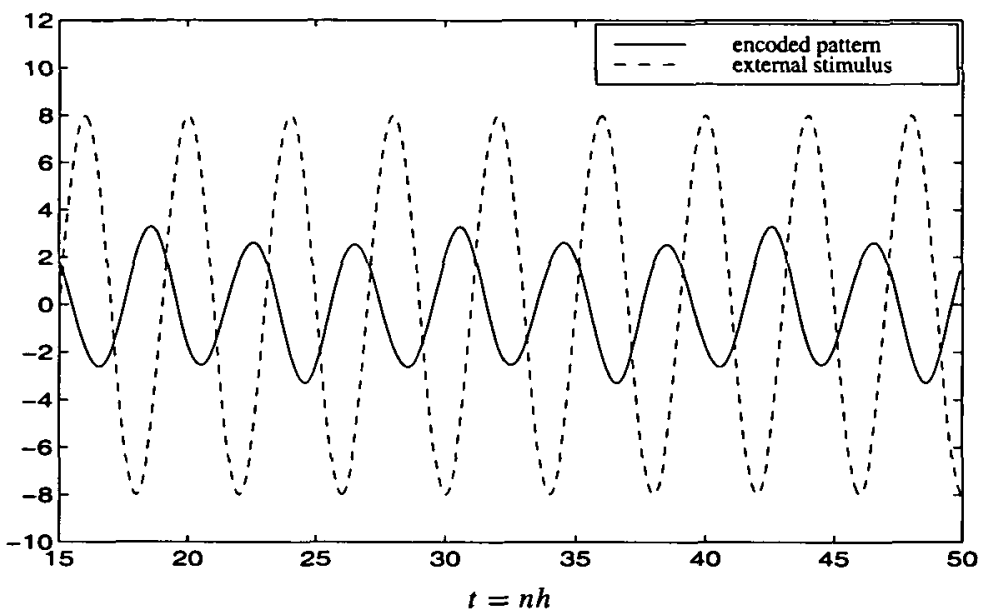

FIGURE 4. The $\omega$-periodic encoded pattern $x_{\omega}^{*}(t)$ associated with the external stimulus $c(t)$ is illustrated for time $15 \leq t \leq 50$.

\section{Discrete-time-continuous-state model}

When one formulates a discrete-time analogue of a model whose characteristics are known from its continuous-time version, it is necessary to derive a discrete-time model which will inherit the dynamical characteristics of the original mother version. The discrete-time models are often used in numerical solutions and computer simulations. One may refer to Mickens [24], Stuart and Humphries [29] and Broomhead and Iserles [4] for various discussions on the importance of discrete-time models in preserving the dynamics of the associated continuous-time systems.

In this section we obtain a discrete-time analogue of the continuous-time model (2.1) and we shall show that the discrete-time analogue preserves the extreme stability conditions of (2.1). We first approximate the continuous-time model (2.1) by an equation with piecewise constant arguments of the form

$$
\begin{aligned}
\frac{d x(t)}{d t}= & -a\left(\left[\frac{t}{h}\right] h\right) x(t)+b\left(\left[\frac{t}{h}\right] h\right) \tanh \left[\sum_{\left[\frac{s}{h}\right]=1}^{\left[\frac{z}{h}\right]} \theta(h) K\left(\left[\frac{s}{h}\right] h\right) x\left(\left[\frac{t}{h}\right] h-\left[\frac{s}{h}\right] h\right)\right] \\
& +c\left(\left[\frac{t}{h}\right] h\right)
\end{aligned}
$$

for $t \in[n h,(n+1) h), n \geq n_{0}, n_{0} \in \mathbb{Z}=\{\ldots,-2,-1,0,1,2, \ldots\}, s \in[j h,(j+1) h)$, $j \in \mathbb{Z}^{+}=\{1,2, \ldots\}$. We note that the interval $n \geq n_{0}$ denotes $n \in\left\{n_{0}, n_{0}+1, \ldots\right\}$. In (3.1), $h>0$ denotes a uniform discretization step-size, $[r]$ denotes the integer part of the real number $r$, and $\theta(h)>0$ for $h>0$ and $\theta(h) \approx h+O\left(h^{2}\right)$ for $h$ small. We 
assume that

$$
\int_{0}^{\tau} K(s) d s \approx \sum_{\left[\frac{s}{h}\right]=1}^{\left[\frac{\tau}{h}\right]} \theta(h) K\left(\left[\frac{s}{h}\right] h\right) \text { for } h>0 .
$$

For convenience, we let $\tilde{K}\left(\left[\frac{s}{h}\right] h\right)=\theta(h) K\left(\left[\frac{s}{h}\right] h\right)$. By applying a semi-implicit Euler-type scheme to (3.1) we obtain

$$
\begin{aligned}
& \frac{x\left(\left[\frac{t}{h}\right] h+h\right)-x\left(\left[\frac{t}{h}\right] h\right)}{h} \\
& =-a\left(\left[\frac{t}{h}\right] h\right) x\left(\left[\frac{t}{h}\right] h+h\right) \\
& +b\left(\left[\frac{t}{h}\right] h\right) \tanh \left[\sum_{\left[\frac{t}{h}\right]=1}^{\left[\frac{x}{h}\right]} \tilde{K}\left(\left[\frac{s}{h}\right] h\right) x\left(\left[\frac{t}{h}\right] h-\left[\frac{s}{h}\right] h\right)\right]+c\left(\left[\frac{t}{h}\right] h\right)
\end{aligned}
$$

for $t \in[n h,(n+1) h), n \geq n_{0}$ and after some simplification we obtain a discretetime-continuous-state model given by

$$
\begin{aligned}
x(n+1)= & \frac{1}{1+a(n) h} x(n)+\frac{b(n) h}{1+a(n) h} \tanh \left[\sum_{j=1}^{\kappa} \tilde{K}(j) x(n-j)\right] \\
& +\frac{c(n) h}{1+a(n) h}
\end{aligned}
$$

for $n \geq n_{0}$ where $\left[\frac{t}{h}\right]=n,\left[\frac{\tau}{h}\right]=\kappa, \kappa \in\{0,1,2, \ldots\}$ and $f(n)=f(n h)$. Since $\theta(h)$ is positive and $K(\cdot)$ nonnegative we have $\tilde{K}(j) \in[0, \infty)$ for $j \in\{1,2, \ldots, \kappa\}$ and $0<\sum_{j=1}^{\kappa} \tilde{K}(j)<\infty$. The neuronal parameters $a(n), b(n)$ and $c(n)$ are sequences of real numbers defined for $n \in \mathbb{Z}$. As a consequence of the assumption (2.2), the parameters satisfy

$$
0<a_{*} \leq a(n) \leq a^{*}, \quad b_{*} \leq|b(n)| \leq b^{*}, \quad c_{*} \leq|c(n)| \leq c^{*}, \quad n \in \mathbb{Z}
$$

where

$$
\begin{aligned}
a_{*}=\inf _{n \in \mathbf{Z}} a(n), & b_{*}=\inf _{n \in \mathbf{Z}}|b(n)|, & c_{*}=\inf _{n \in \mathbf{Z}}|c(n)| \\
a^{*}=\sup _{n \in \mathbf{Z}} a(n), & b^{*}=\sup _{n \in \mathbf{Z}}|b(n)|, & c^{*}=\sup _{n \in \mathbf{Z}}|c(n)| .
\end{aligned}
$$

The initial value for the membrane potential $x(\cdot)$ of $(3.2)$ is given by

$$
x(j)=\varphi(j) \quad \text { for } j \in\left[n_{0}-\kappa, n_{0}\right] \text { and } \sup _{j \in\left[n_{0}-\kappa, n_{0}\right]}|\varphi(j)|<\infty
$$


where $j \in\left[n_{0}-\kappa, n_{0}\right]$ denotes $j \in\left\{n_{0}-\kappa, n_{0}-\kappa+1, \ldots, n_{0}\right\}$. In the following we briefly show the boundedness of solutions satisfying (3.2) and (3.4). By using (3.3) in (3.2) we obtain

$$
|x(n)| \leq \frac{1}{1+a_{*} h}|x(n-1)|+\frac{h\left(b^{*}+c^{*}\right)}{1+a_{*} h} \text { for } n>n_{0}
$$

and from which we derive

$$
|x(n)| \leq \frac{b^{*}+c^{*}}{a_{*}}+\left(\frac{1}{1+a_{*} h}\right)^{n-n_{0}}\left[\left|x\left(n_{0}\right)\right|-\frac{b^{*}+c^{*}}{a_{*}}\right] \text { for } n>n_{0} .
$$

One can easily see from (3.5) that

$$
x\left(n_{0}\right) \in\left[-\frac{b^{*}+c^{*}}{a_{*}}, \frac{b^{*}+c^{*}}{a_{*}}\right] \Longrightarrow x(n) \in\left[-\frac{b^{*}+c^{*}}{a_{*}}, \frac{b^{*}+c^{*}}{a_{*}}\right]
$$

for $n>n_{0}$ and

$$
|x(n)| \leq \frac{b^{*}+c^{*}}{a_{*}}+\gamma(n) \quad \text { for } n>n_{0} \quad \text { and } \quad \gamma(n) \rightarrow 0 \quad \text { as } n \rightarrow \infty .
$$

Let $x(n)=x(n, \varphi)$ and $y(n)=y(n, \psi)$ for $n>n_{0}$ denote arbitrary solutions of (3.2), where $\varphi=\varphi(j)$ and $\psi=\psi(j)$ for $j \in\left[n_{0}-\kappa, n_{0}\right]$. We assume that

$$
\sup _{j \in\left[n_{0}-\kappa, n_{0}\right]}|\varphi(j)-\psi(j)|=M, \quad M>0 .
$$

THEOREM 3.1. Let $h>0$. Suppose (3.3) holds. If there exists a real number $\mu>0$ such that

$$
a(n)-|b(n)| \sum_{j=1}^{\kappa} \tilde{K}(j) \geq \mu \text { for } n \in \mathbb{Z},
$$

then (3.2) is extremely stable in the sense that $\lim _{n \rightarrow \infty}|x(n)-y(n)|=0$, where $x(n)$ and $y(n)$ denote arbitrary solutions of (3.2).

PROOF. Since $x(n)=x(n, \varphi)$ and $y(n)=y(n, \psi)$ are solutions of $(3.2)$ for $n>n_{0}$, we have

$$
\begin{aligned}
x(n+1)-y(n+1)= & \frac{x(n)-y(n)}{1+a(n) h}+\frac{b(n) h}{1+a(n) h} \tanh \left[\sum_{j=1}^{\kappa} \tilde{K}(j) x(n-j)\right] \\
& -\frac{b(n) h}{1+a(n) h} \tanh \left[\sum_{j=1}^{\kappa} \tilde{K}(j) y(n-j)\right]
\end{aligned}
$$


for $n \geq n_{0}$. By applying the mean value theorem of differential calculus we derive from (3.10) that

$$
\begin{aligned}
|x(n+1)-y(n+1)| \leq & \frac{1}{1+a(n) h}|x(n)-y(n)| \\
& +\frac{|b(n)| h}{1+a(n) h} \sum_{j=1}^{\kappa} \tilde{K}(j)|x(n-j)-y(n-j)|
\end{aligned}
$$

for $n \geq n_{0}$ which leads to

$$
v(n+1) \leq \frac{1}{1+a(n) h} v(n)+\frac{|b(n)| h}{1+a(n) h}\left(\sum_{j=1}^{\kappa} \tilde{K}(j)\right) \sup _{n-\kappa \leq m \leq n-1} v(m)
$$

for $n \geq n_{0}$ where $v(\cdot)=|x(\cdot)-y(\cdot)|$.

We gather from (3.8) that $v(n) \leq M$ for $n \in\left[n_{0}-\kappa, n_{0}\right]$. We claim

$$
v(n) \leq M \text { for } n>n_{0} .
$$

Suppose (3.12) is not true. Let there be the first integer $n_{1}$ satisfying $n_{1}>n_{0}$ such that

$$
v(n) \leq M \quad \text { for } n_{0}-\kappa \leq n<n_{1} \text { and } v\left(n_{1}\right)>M .
$$

From (3.11) we obtain

$$
\begin{aligned}
M<v\left(n_{1}\right) & \leq \frac{v\left(n_{1}-1\right)}{1+a\left(n_{1}-1\right) h}+\frac{\left|b\left(n_{1}-1\right)\right| h \sum_{j=1}^{\kappa} \tilde{K}(j)}{1+a\left(n_{1}-1\right) h} \sup _{n_{1}-1-\kappa \leq m \leq n_{1}-2} v(m) \\
& \leq\left(\frac{1+\left|b\left(n_{1}-1\right)\right| h \sum_{j=1}^{\kappa} \tilde{K}(j)}{1+a\left(n_{1}-1\right) h}\right) M .
\end{aligned}
$$

We note that $a(n)$ and $b(n)$ are defined on $\mathbb{Z}$. By using (3.3) and (3.9) we show the following:

$$
\begin{aligned}
0<\frac{1+|b(n)| h \sum_{j=1}^{\kappa} \tilde{K}(j)}{1+a(n) h} & =\frac{1+|b(n)| h \sum_{j=1}^{\kappa} \tilde{K}(j)}{1+a(n) h}-\frac{1+a(n) h}{1+a(n) h}+1 \\
& =-\frac{h}{1+a(n) h}\left(a(n)-|b(n)| \sum_{j=1}^{\kappa} \tilde{K}(j)\right)+1 \\
& \leq-\frac{h \mu}{1+a^{*} h}+1=5 \quad \text { (say) }
\end{aligned}
$$

for $n \in \mathbb{Z}$. Because $h>0$ and $a^{*}>\mu>0$, we have $0<5<1$. Using (3.15) in (3.14) we are led to $M<v\left(n_{1}\right) \leq \varsigma M$ which means a contradiction. Hence the claim (3.12) holds. 
Since $0 \leq v(n) \leq M$ for $n>n_{0}$, there exists a real constant $\lambda$ satisfying $0 \leq \lambda \leq M$ such that

$$
\limsup _{n \rightarrow \infty} v(n)=\lambda
$$

Moreover, since $n-\kappa \rightarrow \infty$ as $n \rightarrow \infty$ we also have

$$
\limsup _{n \rightarrow \infty}\left\{\sup _{n-\kappa \leq m \leq n-1} v(m)\right\}=\lambda \text {. }
$$

In the following we wish to show that $\lambda=0$. Suppose $\lambda>0$. By using the properties of limit superiors, we have from (3.16) and (3.17) that for any $\xi$ satisfying $0<\xi \leq(1-\varsigma) \lambda /(1+\varsigma)$ where $\varsigma$ is given in (3.15), there corresponds a sufficiently large positive integer $N=N(\xi)>n_{0}$ such that

$$
v(n)<\lambda+\xi, \quad \sup _{n-\kappa \leq m \leq n-1} v(m)<\lambda+\xi \quad \text { for } n \geq N .
$$

We gather from (3.11) and (3.18) that

$$
\begin{aligned}
v(n+1) & \leq \frac{1}{1+a(n) h} v(n)+\frac{|b(n)| h}{1+a(n) h}\left(\sum_{j=1}^{\kappa} \tilde{K}(j)\right) \sup _{n-\kappa \leq m \leq n-1} v(m) \\
& <\frac{1+|b(n)| h \sum_{j=1}^{\kappa} \tilde{K}(j)}{1+a(n) h}(\lambda+\xi)
\end{aligned}
$$

for $n \geq N$. Moreover from (3.16), there exists a sequence $\left\{n_{j}\right\}, j \in \mathbb{Z}^{+}$of nonnegative integers with $n_{j} \rightarrow \infty$ as $j \rightarrow \infty$ such that for the same $\xi$ as chosen in the above, there corresponds a positive integer $N_{1}=N_{1}(\xi)$ such that

$$
\lambda-\xi<v\left(n_{j}\right) \text { for all } n_{j} \geq N_{1} .
$$

It follows from (3.19) and (3.20) that

$$
\lambda-\xi<v\left(n_{j}\right)<\varsigma(\lambda+\xi) \text { for all } n_{j} \geq N^{\prime}
$$

where $N^{\prime}=\max \left\{N, N_{1}\right\}$. From (3.21) we obtain that $\xi>(1-\varsigma) \lambda /(1+\varsigma)$ and this contradicts our choice of $\xi$. Thus $\lambda=0$ and hence $\lim _{n \rightarrow \infty}|x(n)-y(n)|=0$. This completes the proof.

\section{Some remarks}

We have obtained sufficient conditions under which the continuous-time neuronal model is extremely stable. The same conditions guarantee the model to have heteroassociative stable encoding of temporally non-uniform stimuli. In this study we have assumed that the neuron oscillates synchronously in response to the external input 
stimulus. If the external stimulus is of almost periodic type (or periodic type) then under the sufficient conditions the resulting encoded pattern (a global attractor) is almost periodic (or periodic).

Throughout the study we have incorporated delays distributed over a finite time interval in the processing part of the neuron's architecture. We note that one can apply the same analysis to a model with finite delays given in the form

$$
\frac{d x(t)}{d t}=-a(t) x(t)+b(t) \tanh [x(t-\tau)]+c(t), \quad t>t_{0}
$$

and the associated discrete-time formulation in the form

$$
x(n+1)=\frac{1}{1+a(n) h} x(n)+\frac{b(n) h}{1+a(n) h} \tanh [x(n-\kappa)]+\frac{c(n) h}{1+a(n) h}
$$

for $n \geq n_{0}$. One then obtains that if

$$
\begin{aligned}
& a(t)-b(t) \geq v, \quad t \in \mathbb{R} \quad \text { (continuous) } \\
& a(n)-b(n) \geq \nu, \quad n \in \mathbb{Z} \quad \text { (dsicrete) }
\end{aligned}
$$

for some $v>0$ then the hetero-associative stable recall of the encoded pattern is guaranteed in both models (4.1) and (4.2). One observes from (4.3) that the discrete delays in (4.1) and (4.2) do not affect the neuron's capability to encode the external stimulus and recall the encoded pattern.

In this article we have systematically formulated a discrete-time model based on discretizing the respective continuous-time equation. We have seen that discretization step-size $h>0$ does not express itself explicitly in the stability conditions. Moreover, the extreme stability conditions of the continuous-time system are preserved by the discrete-time model. These advantages inherited in the discrete-time model are lacking in some conventional numerical schemes like the Euler-type and Runge-Kutta-type schemes. In fact, one can employ the same analysis as shown in this article on the Euler-type scheme and show how the step-size $h>0$ restricts the range of values of the parameter $a(\cdot)$ (see also Wang and Blum [30]).

Acknowledgement The authors wish to thank the referees for their suggestions which have improved the presentation of the results.

\section{References}

[1] C. T. H. Baker and A. Tang, "Generalised Halanay inequalities for Volterra functional differential equations and discretised versions", Report 299, Manchester Centre for Computational Mathematics, The University of Manchester, England, 1996. 
[2] A. S. Besicovitch, Almost Periodic Functions (Dover, New York, 1954).

[3] V. E. Bondarenko, "Self-organization processes in chaotic neural networks under external periodic force", Int. J. Bifur. Chaos 7 (1997) 1887-1895.

[4] D. S. Broomhead and A. Iserles (eds.), The dynamics of numerics and the numerics of dynamics (Clarendon Press, Oxford, 1992).

[5] E. R. Caianiello and de Luca, "Decision equation for binary systems; application to neuronal behaviour", Kybernetik 3 (1966) 33-40.

[6] F. Chapeau-Blondeau and G. Chauvet, "Stable, oscillatory and chaotic regimes in the dynamics of small neural networks with delay", Neural Networks 5 (1992) 735-744.

[7] C. Corduneanu, Almost Periodic Functions (Interscience, New York, 1968).

[8] R. Eckhorn, R. Bauer, W. Jordan, W. Kruse, M. Munk and H. J. Rertboeck, "Coherent oscillations: A mechanism for feature linking in the visual cortex", Biol. Cybern. 60 (1988) 121-130.

[9] A. K. Engel, A. K. Kreiter, P. König and W. Singer, "Synchronization of oscillatory neural responses between striate and extrastriate visual cortical areas of the cat", Proc. Natl. Acad. Sci. 88 (1991) 6048-6052.

[10] A. M. Fink, Almost Periodic Differential Equations (Springer, New York, 1974).

[11] W. J. Freeman, "The physiology of perception", Sci. Amer. February (1991).

[12] W. J. Freeman, Y. Yau and B. Burke, "Central pattern generating and recognizing in olfactory bulb: A correlation learning rule", Neural Networks 1 (1988) 277-288.

[13] K. Gopalsamy, Stability and Oscillations in Delay Differential Equations of Population Dynamics (Kluwer Academic Publishers, The Netherlands, 1992).

[14] K. Gopalsamy and X. Z. He, "Delay-independent stability in bidirectional associative memory networks", IEEE Trans. Neural Networks 5 (1994) 998-1002.

[15] K. Gopalsamy and X. Z. He, "Stability in asymmetric Hopfield nets with transmission delays", Physica D 76 (1994) 344-358.

[16] K. Gopalsamy and X. Z. He, "Dynamics of an almost periodic logistic integrodifferential equation", Methods Appl. Anal. 2 (1995) 38-66.

[17] K. Gopalsamy, X. Z. He and L. Wen, "Global attractivity and oscillations in an almost periodic delay logistic equation", Nonlinear Times Digest 1 (1994) 9-24.

[18] C. M. Gray, P. König, A. K. Engel and W. Singer, "Oscillatory responses in cat visual cortex exhibit intercolumnar synchronization which reflects global stimulus properties", Nature 388 (1989) 334 337.

[19] A. Halanay, Differential Equations (Academic Press, New York, 1966).

[20] A. Hjelmfelt and J. Ross, "Pattern recognition, chaos and multiplicity in neural networks and excitable systems", Proc. Nat. Academy Sci. 91 (1994) 63-67.

[21] V. I. Istratescu, Fixed Point Theory (D. Riedel Publ. Co., Dordrecht, 1981).

[22] P. König and J. B. Schillen, "Stimulus-dependent assembly formation of oscillatory responses: I. Synchronization", Neural Comput. 3 (1991) 155-166.

[23] L. P. LaSalle and S. Lefschetz, Stability by Lyapunov's Direct Method with Applications (Academic Press, New York, 1961).

[24] R. E. Mickens, Nonstandard Finite Difference Models of Differential Equations (World Scientific, River Edge, NJ, 1994).

[25] J. E. Moreira and D. M. Auto, "Intermittency in a neural network with variable threshold", Europhys. Lett. 21 (1993) 639.

[26] E. Ott, C. Grebogi and J. A. Yorke, "Controlling chaos", Phys. Rev. Lett. 64 (1990) 1196-1199.

[27] A. Rescigno, R. B. Stein, R. L. Stein and R. E. Poppele, "A neuronal model for the discharge patterns produced by cyclic inputs", Bull. Math. Biophys. 32 (1970) 337-353.

[28] C. A. Skarda and W. J. Freeman, "How brains make chaos in order to make sense of the world", Brain Behavioural Sci. 10 (1987) 161-195. 
[29] A. M. Stuart and A. R. Humphries, Dynamical systems and numerical analysis (Cambridge University Press, Cambridge, 1996).

[30] X. Wang and E. K. Blum, "Discrete-time versus continuous-time models of neural networks", $J$. Comput. Syst. Sci. 45 (1992) 1-19.

[31] Y. Yau, W. J. Freeman, B. Burke and Q. Yang, "Pattern recognition by a distributed neural network: An industrial application", Neural Networks 4 (1991) 103-121.

[32] T. Yoshizawa, "Extreme stability and almost periodic solutions of functional-differential equations", Arch. Rat. Mech. Anal. 17 (1964) 148-170.

[33] T. Yoshizawa, Stability Theory and the Existence of Periodic Solutions and Almost Periodic Solutions (Springer, New York, 1975). 